\title{
Observer and Stochastic Faulty Actuator-Based Reliable Consensus Protocol for Multiagent System
}

\author{
Rathinasamy Sakthivel ${ }^{\circledR}$, Boomipalagan Kaviarasan, Choon Ki Ahn ${ }^{\circledR}$, Senior Member, IEEE, \\ and Hamid Reza Karimi ${ }^{\circledR}$, Senior Member, IEEE
}

\begin{abstract}
A nondeterministic actuator fault model-based reliable control design method is presented for the leaderfollowing consensus of a multiagent system (MAS) subject to input time-varying delay and the nonlinear phenomenon through the observer framework. Notably, a more generalized practical actuator fault model is proposed where the faults of each actuator are assumed to occur randomly and the fault rates are characterized by stochastic variables that satisfy certain probability conditions. To trace the leader dynamics, a reliable observerbased state feedback consensus algorithm is developed for the pursuing agents. After that, in light of graph theory and the Lyapunov-Krasovskii stability theorem, the required conditions are achieved to guarantee the leader-following consensus of the considered MAS, and then the corresponding reliable controller design method is proposed. Furthermore, simulations are eventually conducted to exhibit the efficiency of the newly developed control scheme, where it is shown that the obtained conditions guarantee the consensus of the considered MAS in the case of possible actuator faults.
\end{abstract}

Index Terms-Actuator faults, leader-following consensus, multiagent system (MAS), stochastic reliable control.

\section{INTRODUCTION}

$\mathbf{M}$ ULTIAGENT systems (MASs) are composed of a group of interacting agents that interact mutually with one another using particular mechanisms and protocols. Since MASs accomplish crucial tasks in a coordinated manner, they have several benefits compared with conventional control systems, such as minimizing total expenditure, providing a better level of system performance, and providing new capabilities. Meanwhile, consensus is the most significant and prominent property in the study of MASs, which means that

Manuscript received June 19, 2017; accepted September 18, 2017. Date of publication October 17, 2017; date of current version November 15, 2018 This paper was recommended by Associate Editor Y.-J. Liu. (Corresponding author: Choon Ki Ahn.)

R. Sakthivel is with the Department of Mathematics, Bharathiar University, Coimbatore 641046, India, and also with the Department of Mathematics, Sungkyunkwan University, Suwon 440-746, South Korea (e-mail: krsakthivel@yahoo.com).

B. Kaviarasan is with the Department of Mathematics, Anna University Regional Campus, Coimbatore 641046, India (e-mail: bkavi1000@gmail.com).

C. K. Ahn is with the School of Electrical Engineering, Korea University, Seoul 136-701, South Korea (e-mail: hironaka@korea.ac.kr).

H. R. Karimi is with the Department of Mechanical Engineering, Politecnico di Milano, 20156 Milan, Italy (e-mail: hamidreza.karimi@polimi.it).

Color versions of one or more of the figures in this paper are available online. all the interacting agents will eventually arrive at a desired fixed value [1]-[4], [6]. In practice, the consensus of MASs has wide applications in numerous areas, such as wireless sensor networks, formation cooperative control problems, and the scheduling of automated highway systems [5], [7]; thus, the investigation of the consensus of MASs has received a great deal of attention among research communities during the last few decades [8]-[12].

In the context of consensus problems, an interesting and important area is the leader-following consensus, where there exists an agent acting as a leader and the states of all agents are required to reach the state of the leader [13]. Additionally, in the perspective of applications, the leader-following consensus has received considerable interest due to its significant usages in diverse systems, such as vehicle systems, intelligent decision support systems for power grid dispatching, and networked control systems. So far in the literature, many interesting problems have been studied regarding the leaderfollowing consensus with different agent dynamics, including first-order, second-order, and high-order systems [14]-[17]. Furthermore, nonlinearity exists in practical MASs; thus, interesting results on the leader-following consensus have been obtained for nonlinear MASs in [18] and [19].

The aforementioned leader-following consensus algorithms have predominantly assumed that the leader dynamics are known or fixed. Unfortunately, this assumption does not always hold in real-world situations because the leader dynamics may be an unfixed reference and known only partially [20]. Furthermore, it is uncertain whether the leader's command signal can be accessed by the tracking agents when the number of agents is too high. In such critical situations, the distributed observer-based technique is generally employed in the algorithm design to track the unknown or unfixed signal [21]. Numerous interesting results on observer-based problems have been reported in [22]-[30]. Following these seminal works, in [31], based on centralized and distributed event-triggered mechanisms, the consensus problem of linear MASs was studied under observer-based control protocols. Additionally, a novel distributed consensus algorithm for both discrete and continuous-time MASs was developed in [32] under the observer framework. Recently, by employing the concepts from system control theory and algebraic graph theory, the consensus issue of general linear MASs was solved in [33] by using the leader-follower framework, where two different functional observers are adopted in distributed consensus protocols to estimate the unknown inputs. However, to 
date, very few results have been published regarding the issue of the leader-following consensus design for MASs through the observer approach, which is the main motivation of this paper.

On the other hand, a major concern of control communities is the possible failure of an actuator during the task of control signal transmission because faulty actuators may deteriorate the control system performance or even cause potential damage [34]. Therefore, the control system requires a sophisticated control strategy to ensure and maintain desirable performance even in the presence of faulty actuators. Owing to this requirement, control communities have developed a control strategy called reliable control whose aim is to maintain the desirable levels and stability conditions of the control system not only when the control components are working in normal conditions, but also in the existence of some possible actuator faults [35]. Consequently, research on reliable control consisting of actuator faults has drawn a remarkable amount of attention (e.g., [36]-[41]) and is of great importance from both theoretical and application-based perspectives. Following these seminal works, reliable control problems for the consensus of MASs have been eagerly examined in the past few years [42], [43]. The aforementioned research on reliable control has mainly considered two cases: 1) when the actuator fault is a fixed constant and 2) when the actuator fault is an unfixed value, but lies in a bounded interval. However, in practical engineering problems, the actuator fault may occur in a stochastic manner because the patterns and modes of actuator faults are essentially random in nature, so the cases considered above cannot represent actuator faults exactly. Thus, it is more applicable and meaningful to consider a fault factor that obeys a certain probabilistic distribution in an interval. Based on this scenario, only very few results have been obtained in [44] and [45]. To date and to the best of our knowledge, the problem of leader-following consensus of nonlinear MASs by using a reliable controller with stochastic faults has not been yet completely addressed in the literature, which partly motivates us for the current study.

By employing the concepts from the aforementioned MAS works, in this paper, we will design an observer-based reliable controller subject to stochastic actuator faults that confirms the leader-following consensus of nonlinear MASs. First, a reliable state feedback consensus algorithm is proposed based on the observer technique, where the concept of stochastic actuator faults is taken into account. Second, by using simple mathematical transformations, two types of error systems are formulated. Third, based on the Lyapunov stability theorem [47], sufficient conditions guaranteeing the asymptotic stability of resulting error systems are derived in the meansquare sense, which ensures the leader-following consensus of the considered MAS. Subsequently, the observer and state feedback gain parameters are estimated. Then, to show the potential of the newly proposed control design, a simulation example is provided.

The novelty and significant advantages of this paper are as follows.

1) This paper is the first attempt to discuss the issue of the leader-following consensus of the nonlinear MAS under an observer-based reliable protocol subject to probabilistic actuator faults and a switching topology. Compared with the existing works on linear MASs, the results obtained in this paper are more applicable, since most MASs are, in practice, nonlinear. Moreover, the actuator faults during the control design are assumed to occur in a random manner such that the considered model in this paper is more realistic.

2) Sufficient conditions guaranteeing the asymptotic stability of the resulting error systems are established in the mean-square sense for the achievement of the leader-following consensus of nonlinear MASs. Then, the desired observer and reliable state feedback gain matrices are obtained for a solution to linear matrix inequality (LMI) constraints.

3) The results developed in this paper satisfy both normal and faulty actuator cases; that is, all the actuators work in the normal mode and some of the actuators work in the faulty mode, which reveals that our results are more general and practical.

\section{GRAPH THEORY PRELIMINARIES AND MODEL DESCRIPTION}

Here, we first review some useful graph theory concepts, and then the problem of the leader-following consensus of nonlinear input-delayed MASs will be presented.

In this paper, to represent the interactions among the MAS agents, an undirected graph $\mathcal{G}=(\mathcal{V}, \mathcal{E}, \mathcal{W})$ of order $N$ is chosen, where $\mathcal{V}=\left\{v_{1}, v_{2}, \ldots, v_{N}\right\}$ denotes the set of all agents, $\mathcal{E} \subseteq \mathcal{V} \times \mathcal{V}$ represents the edges' set, and $\mathcal{W}=\left[a_{i j}\right]_{N \times N}$ is the adjacency matrix with $a_{i j}>0$ if $\left(v_{i}, v_{j}\right) \in \mathcal{E}$ and $a_{i j}=0$ otherwise. Moreover, the neighbor set of $v_{i}$ can be represented by $\mathcal{N}_{i}=\left\{j:\left(v_{i}, v_{j}\right) \in \mathcal{E}\right\}$. Let $\mathcal{D}=\operatorname{diag}\left\{d_{1}, d_{2}, \ldots, d_{N}\right\}$ be the degree matrix of graph $\mathcal{G}$ with entries $d_{i}=\sum_{j \in \mathcal{N}_{i}} a_{i j}$. Then, the Laplacian matrix of $\mathcal{G}$ can be expressed as $L=\mathcal{D}-\mathcal{W}$.

In the context of the leader-following consensus of the MAS, to represent the interactions between the agents and leader, we use the graph $\hat{\mathcal{G}}$ of order $(N+1)$, which contains $N$ following agents (already specified in $\mathcal{G}$ ) and one leader (denoted as $v_{0}$ ). The leader can pass the information to tracking agents, but cannot acquire any information from them. Supposing that there are variable interconnection topologies, we denote the collection of all possible interaction graphs as $\mathbb{G}=\left\{\hat{\mathcal{G}}_{1}, \hat{\mathcal{G}}_{2}, \ldots, \hat{\mathcal{G}}_{M}\right\}$ with the index set $\mathcal{O}=\{1,2, \ldots, M\}$. To represent the index of the topology graph, we use the switching signal $\sigma(t):[0, \infty) \rightarrow \mathcal{O}$. Then, the Laplacian matrix associated with the underlying graph $\hat{\mathcal{G}}_{\sigma(t)}$ is denoted as $L_{\sigma(t)}$.

Consider a continuous-time nonlinear MAS with input timevarying delay that is composed of $N$ identical agents and a leader. Then, the motion of the agents' state can be governed by the following equations:

$$
\begin{aligned}
& \dot{x}_{i}(t)=A x_{i}(t)+B u_{i}^{f}(t-\varepsilon(t))+g\left(x_{i}(t), t\right) \\
& y_{i}(t)=C x_{i}(t), \quad i=1,2, \ldots, N \\
& x_{i}(t)=\phi(t), \quad t \in\left[-\varepsilon_{2}, 0\right]
\end{aligned}
$$


where $x_{i} \in \mathbb{R}^{n}$ and $u_{i}^{f} \in \mathbb{R}^{m}$ are the $i$ th agent's state and input with possible faults, respectively; $y_{i} \in \mathbb{R}^{q}$ is the measured output of the $i$ th agent; $\varepsilon(t)$ is the input delay satisfying $0<$ $\varepsilon_{1} \leq \varepsilon(t) \leq \varepsilon_{2}$ and $\dot{\varepsilon}(t) \leq \lambda<1$; and $A, B$, and $C$ are real constant matrices. Furthermore, $\phi(t)$ is the initial value of the $i$ th agent's state.

Additionally, the motion of the leader's state can be expressed as follows:

$$
\begin{aligned}
& \dot{x}_{0}(t)=A x_{0}(t)+g\left(x_{0}(t), t\right) \\
& y_{0}(t)=C x_{0}(t)
\end{aligned}
$$

where $x_{0} \in \mathbb{R}^{n}$ and $y_{0} \in \mathbb{R}^{q}$ are the state and measured output of the leader, respectively.

It can be observed from the existing literature that most of the results on the leader-following consensus have considered the leader's dynamics fixed. However, this is not the case in some practical situations because the leader's dynamics may vary for certain reasons, which are specified in Section I. In these situations, to trace the states of the leader, an observerbased protocol is essential. Thus, the observer-based dynamics for the MAS (1) in question can be chosen in the following manner:

$$
\begin{aligned}
\dot{\hat{x}}_{i}(t)= & A \hat{x}_{i}(t)+g\left(\hat{x}_{i}(t), t\right)-D \sum_{j \in \mathcal{N}_{i}} \alpha_{i j}(t)\left[\hat{y}_{i}(t)-\hat{y}_{j}(t)\right] \\
& -D \beta_{i}(t)\left[\hat{y}_{i}(t)-y_{0}(t)\right] \\
\hat{y}_{i}(t)= & C \hat{x}_{i}(t), \quad i=1,2, \ldots, N
\end{aligned}
$$

where $\hat{x}_{i} \in \mathbb{R}^{n}$ is the observer state, $D \in \mathbb{R}^{n \times q}$ is the observer gain matrix, and the parameters $\alpha_{i j}(t)$ and $\beta_{i}(t)$ are defined by

$$
\begin{aligned}
\alpha_{i j}(t) & = \begin{cases}a_{i j}, & \text { if agents } i \text { and } j \text { have connection } \\
0, & \text { else }\end{cases} \\
\beta_{i}(t) & = \begin{cases}b_{i}, & \text { if agent } i \text { has connection with leader } \\
0, & \text { else }\end{cases}
\end{aligned}
$$

where $a_{i j}>0$ are the connection weight constants between agents $i$ and $j$ and $b_{i}>0$ are the connection weight constants between agent $i$ and the leader.

Throughout this paper, we assume the following two conditions: 1) the nonlinear vector-valued function $g(x(t), t)$ satisfies $\left\|g\left(x_{i}(t), t\right)\right\| \leq W_{1}\left\|x_{i}(t)\right\|$, where $W_{1}>0$ is a diagonal matrix and $x_{i}(t) \in \mathbb{R}^{n}$ and 2) matrix $C$ has rank $q$. For representation purposes, the singular value decomposition of $C$ is denoted as $C=U\left[\begin{array}{ll}S & 0\end{array}\right] V^{\mathrm{T}}$, where $U \in \mathbb{R}^{q \times q}$ and $V \in \mathbb{R}^{n \times n}$ are unitary matrices and $S \in \mathbb{R}^{q \times q}$ is a diagonal matrix with positive entries in decreasing order.

Now it is time to propose the control law to achieve the leader-following consensus of the nonlinear MAS (1). As discussed earlier, there may be plenty of reasons for actuator faults during the control signal transmission. So far in the literature, various kinds of actuator fault models have been introduced in the control design [34]-[36], [39], [44], [45]. Among them, the actuator fault model consisting of stochastic faults is more applicable in practice and covers the existing fault models [46]. Therefore, in this paper, the control law with stochastic faults for (1) can be proposed as follows:

$$
u_{i}^{f}(t-\varepsilon(t))=H u_{i}(t-\varepsilon(t))=\sum_{k=1}^{m} \delta_{k} E_{k} u_{i}(t-\varepsilon(t))
$$

where $H=\operatorname{diag}\left\{\delta_{1}, \delta_{2}, \ldots, \delta_{m}\right\}$ is the actuator fault matrix and $\delta_{k}(k=1,2, \ldots, m)$ are the stochastic variables, which take values in the interval $[0,1] ; E_{k}=\operatorname{diag}\{\underbrace{0, \ldots, 0}_{k-1}, 1, \underbrace{0, \ldots, 0}_{m-k}\}$ $(k=1,2, \ldots, m)$. Additionally, the delayed-state feedback controller is defined by $u_{i}(t-\varepsilon(t))=K\left[\hat{x}_{i}(t-\varepsilon(t))-x_{i}(t-\right.$ $\varepsilon(t))$ ], where $K$ is the feedback gain matrix to be determined. Since $\delta_{k}$ s are stochastic variables, the mean and variance of $\delta_{k} \mathrm{~s}$ are denoted as $\varpi_{k}$ and $\vartheta_{k}^{2}$, respectively. Moreover, by letting $\mathbb{E}\{H\}=\bar{H}$, it is easy to obtain $\mathbb{E}\{H-\bar{H}\}=0$ and $\mathbb{E}\{(H-$ $\left.\bar{H})^{\mathrm{T}} P(H-\bar{H})\right\}=\sum_{k=1}^{m} \vartheta_{k}^{2} E_{k}^{\mathrm{T}} P E_{k}$, where $P>0$.

Remark 1: Motivated by the works in [44] and [45], a probability-dependent actuator fault model is adopted during the design of the reliable controller (4) for the nonlinear MAS (1). This fault model includes some of the existing fault models [34], [35] as special cases; thus, it can be treated as a more general fault model. However, this paper is the first attempt in the literature to consider the occurrence of actuator faults in a probabilistic manner for the nonlinear MAS.

Then, by substituting the control term (4) in (1), we can obtain the following equations:

$$
\begin{aligned}
\dot{x}_{i}(t)= & A x_{i}(t)+B \sum_{k=1}^{m} \delta_{k} E_{k} K\left[\hat{x}_{i}(t-\varepsilon(t))-x_{i}(t-\varepsilon(t))\right] \\
& +g\left(x_{i}(t), t\right) \\
y_{i}(t)= & C x_{i}(t), \quad i=1,2, \ldots, N .
\end{aligned}
$$

In what follows, to improve the analysis of the leaderfollowing consensus of the nonlinear MAS (1), the consensus problem is equivalently changed to the stabilization problem of corresponding error systems. For this purpose, we define the consensus error vectors as $\hat{e}_{i}(t)=\hat{x}_{i}(t)-x_{0}(t)$ and $e_{i}(t)=x_{i}(t)-x_{0}(t)$. Then, it is easy to find that

$$
\begin{aligned}
\dot{\hat{e}}_{i}(t)= & A \hat{e}_{i}(t)+G_{1}\left(\hat{e}_{i}(t), t\right) \\
& -D\left(\sum_{j \in \mathcal{N}_{i}} \alpha_{i j}(t) C\left[\hat{e}_{i}(t)-\hat{e}_{j}(t)\right]+\beta_{i}(t) C \hat{e}_{i}(t)\right) \\
\dot{e}_{i}(t)= & A e_{i}(t)-B H K e_{i}(t-\varepsilon(t))+B H K \hat{e}_{i}(t-\varepsilon(t)) \\
& +G_{2}\left(e_{i}(t), t\right)
\end{aligned}
$$

where $G_{1}\left(\hat{e}_{i}(t), t\right)=g\left(\hat{x}_{i}(t), t\right)-g\left(x_{0}(t), t\right)$ and $G_{2}\left(e_{i}(t), t\right)=$ $g\left(x_{i}(t), t\right)-g\left(x_{0}(t), t\right)$.

By utilizing the properties of the Kronecker product and simple manipulation, the above error systems can be rewritten in the following form:

$$
\begin{aligned}
\dot{\hat{e}}(t)= & {\left[(I \otimes A)-\left(\mathcal{L}_{\sigma(t)} \otimes D C\right)\right] \hat{e}(t)+G_{1}(\hat{e}(t), t) } \\
\dot{e}(t)= & (I \otimes A) e(t)-[I \otimes B(H-\bar{H}) K] e(t-\varepsilon(t)) \\
& -(I \otimes B \bar{H} K) e(t-\varepsilon(t)) \\
& +[I \otimes B(H-\bar{H}) K] \hat{e}(t-\varepsilon(t)) \\
& +(I \otimes B \bar{H} K) \hat{e}(t-\varepsilon(t))+G_{2}(e(t), t)
\end{aligned}
$$

where $\hat{e}(t)=\left[\begin{array}{llll}\hat{e}_{1}(t) & \hat{e}_{2}(t) & \cdots & \hat{e}_{N}(t)\end{array}\right]^{\mathrm{T}}, \quad G_{1}(\hat{e}(t), t)=$ $\left[\begin{array}{llll}G_{1}\left(\hat{e}_{1}(t), t\right) & G_{1}\left(\hat{e}_{2}(t), t\right) & \cdots & G_{1}\left(\hat{e}_{N}(t), t\right)\end{array}\right]^{\mathrm{T}}, e(t)=\left[e_{1}(t)\right.$ $\left.e_{2}(t) \cdots e_{N}(t)\right]^{\mathrm{T}}, G_{2}(e(t), t)=\left[G_{2}\left(e_{1}(t), t\right) G_{2}\left(e_{2}(t), t\right) \cdots\right.$ $\left.G_{2}\left(e_{N}(t), t\right)\right]^{\mathrm{T}}$, and $\mathcal{L}_{\sigma(t)}=L_{\sigma(t)}+F_{\sigma(t)}$, and $F_{\sigma(t)}$ is a diagonal matrix of order $N$ whose $i$ th diagonal element is $\beta_{i}(t)$. 
The following lemma is essential to obtain the desired result in the following section.

Lemma 1 [48]: For any given matrix $C \in \mathbb{R}^{q \times n}(q<n)$ with full row rank that is, $\operatorname{rank}(C)=q$ and a symmetric matrix $P$ of order $n$ there exists a matrix $\hat{P}$ of order $n$ satisfying $C P=$ $\hat{P} C$ if and only if $P$ can be described as $P=V\left[\begin{array}{cc}P_{11} & 0 \\ 0 & P_{22}\end{array}\right] V^{\mathrm{T}}$, where $P_{11} \in \mathbb{R}^{q \times q}$ and $P_{22} \in \mathbb{R}^{(n-q) \times(n-q)}$.

\section{LEADER-FOLLOWING CONSENSUS CRITERION FOR NONLINEAR MAS}

In this section, by using a few concepts of algebraic graph theory and the Lyapunov stability theorem, we will establish sufficient conditions that guarantee the leaderfollowing consensus of the MAS (1) and the control design of the probability-based reliable controller (4). Meanwhile, to shorten the derivation part, let us use block entry matrices $z_{i}(i=1,2, \ldots, 18) \in \mathbb{R}^{18 n \times n}$, such as $z_{4}=$ $\left[\begin{array}{llllll}0_{n} & 0_{n} & 0_{n} & I_{n} & \underbrace{0_{n}}_{14} \quad \ldots & 0_{n}\end{array}\right]^{\mathrm{T}}$ and the following notions:

$$
\begin{aligned}
& \psi_{1}=\left[\begin{array}{ll}
z_{1} & z_{9}
\end{array}\right]\left[(I \otimes A)-\left(\mathcal{L}_{\sigma(t)} \otimes D C\right) \quad I\right]^{\mathrm{T}} \\
& \psi_{2}=\left[\begin{array}{llll}
z_{10} & z_{12} & z_{3} & z_{18}
\end{array}\right] \\
& \times\left[\begin{array}{llll}
(I \otimes A) & -(I \otimes B \bar{H} K) & (I \otimes B \bar{H} K) & I
\end{array}\right]^{\mathrm{T}} \\
& \eta_{1}=\left[\begin{array}{llll}
z_{1} & z_{2} & z_{5} & z_{7}
\end{array}\right], \quad \eta_{2}=\left[\begin{array}{llll}
z_{10} & z_{11} & z_{14} & z_{16}
\end{array}\right] \\
& \eta_{3}=\left[\begin{array}{llll}
z_{2} & z_{4} & z_{6} & z_{8}
\end{array}\right], \quad \eta_{4}=\left[\begin{array}{llll}
z_{11} & z_{13} & z_{15} & z_{17}
\end{array}\right] \\
& N=\left[\begin{array}{ccc}
I_{n} & I_{n} & I_{n} \\
-I_{n} & I_{n} & -I_{n} \\
0 & -2 I_{n} & 6 I_{n} \\
0 & 0 & -6 I_{n}
\end{array}\right] \\
& \bar{R}_{a}=\operatorname{diag}\left\{\left(I \otimes R_{a}\right), 3\left(I \otimes R_{a}\right), 5\left(I \otimes R_{a}\right)\right\}(a=5,6) \\
& \Xi_{1}=\left[\begin{array}{ll}
z_{1} & z_{10}
\end{array}\right]\left(I \otimes R_{1}\right)\left[\begin{array}{ll}
\psi_{1} & \psi_{2}
\end{array}\right]^{\mathrm{T}} \\
& +\left[\begin{array}{ll}
\psi_{1} & \psi_{2}
\end{array}\right]\left(I \otimes R_{1}\right)\left[\begin{array}{ll}
z_{1} & z_{10}
\end{array}\right]^{\mathrm{T}} \\
& \Xi_{2}=\left[\begin{array}{ll}
z_{1} & z_{10}
\end{array}\right]\left(\left(I \otimes R_{2}\right)+\left(I \otimes R_{3}\right)\right)\left[\begin{array}{ll}
z_{1} & z_{10}
\end{array}\right]^{\mathrm{T}} \\
& +\left[\begin{array}{ll}
z_{2} & z_{11}
\end{array}\right]\left(\left(I \otimes R_{4}\right)-\left(I \otimes R_{2}\right)\right)\left[\begin{array}{ll}
z_{2} & z_{11}
\end{array}\right]^{\mathrm{T}} \\
& -(1-\lambda)\left[\begin{array}{ll}
z_{3} & z_{12}
\end{array}\right]\left(I \otimes R_{3}\right)\left[\begin{array}{ll}
z_{3} & z_{12}
\end{array}\right]^{\mathrm{T}} \\
& -\left[\begin{array}{ll}
z_{4} & z_{13}
\end{array}\right]\left(I \otimes R_{4}\right)\left[\begin{array}{ll}
z_{3} & z_{13}
\end{array}\right]^{\mathrm{T}} \\
& \Xi_{3}=\Xi_{31}+\Xi_{32}+\Xi_{33}+\Xi_{34}, \quad \Xi_{31}=-\frac{1}{\varepsilon_{1}} \eta_{1} N \bar{R}_{5} N^{\mathrm{T}} \eta_{1}^{\mathrm{T}} \\
& \Xi_{32}=-\frac{1}{\varepsilon_{1}} \eta_{2} N \bar{R}_{5} N^{\mathrm{T}} \eta_{2}^{\mathrm{T}}, \quad \Xi_{33}=-\frac{1}{\varepsilon_{2}-\varepsilon_{1}} \eta_{3} N \bar{R}_{6} N^{\mathrm{T}} \eta_{3}^{\mathrm{T}} \\
& \Xi_{34}=-\frac{1}{\varepsilon_{2}-\varepsilon_{1}} \eta_{4} N \bar{R}_{6} N^{\mathrm{T}} \eta_{4}^{\mathrm{T}} \\
& \Xi_{4}=\left[\begin{array}{ll}
\Xi_{41} & \Xi_{42}
\end{array}\right]\left(I \otimes Q_{1}\right)\left[z_{2}-z_{3} \quad z_{11}-z_{12}\right]^{\mathrm{T}} \\
& +\left[\begin{array}{ll}
\Xi_{41} & \Xi_{42}
\end{array}\right]\left(I \otimes Q_{2}\right)\left[\begin{array}{ll}
z_{3}-z_{4} & z_{12}-z_{13}
\end{array}\right]^{\mathrm{T}} \\
& \Xi_{41}=\left[\begin{array}{llll}
z_{1} & z_{3} & z_{10} & z_{12}
\end{array}\right], \quad \Xi_{42}=\left[\begin{array}{llll}
z_{2} & z_{4} & z_{11} & z_{13}
\end{array}\right] \\
& \Xi_{5}=\left[\begin{array}{ll}
z 9 & z_{18}
\end{array}\right] \operatorname{diag}\{-(I \otimes I),-(I \otimes I)\}\left[\begin{array}{ll}
z_{9} & z_{18}
\end{array}\right]^{\mathrm{T}} \\
& \hat{\psi}_{1}=\left[\begin{array}{ll}
z_{1} & z_{9}
\end{array}\right]\left[(I \otimes A)\left(I \otimes P_{1}\right)-\left(\mathcal{L}_{\sigma(t)} \otimes Y_{1} C\right) \quad I\right]^{\mathrm{T}} \\
& \hat{\psi}_{2}=\left[\begin{array}{llll}
z_{10} & z_{12} & z_{3} & z_{18}
\end{array}\right] \\
& \times\left[(I \otimes A)\left(I \otimes P_{1}\right)-\left(I \otimes B \bar{H} Y_{2}\right)\left(I \otimes B \bar{H} Y_{2}\right) I\right]^{\mathrm{T}} \\
& \hat{\bar{R}}_{a}=\operatorname{diag}\left\{\left(I \otimes \hat{R}_{a}\right), 3\left(I \otimes \hat{R}_{a}\right), 5\left(I \otimes \hat{R}_{a}\right)\right\}(a=5,6)
\end{aligned}
$$

$$
\begin{aligned}
& \hat{\Xi}_{1}=\left[\begin{array}{ll}
z_{1} & z_{10}
\end{array}\right]\left[\begin{array}{ll}
\hat{\psi}_{1} & \hat{\psi}_{2}
\end{array}\right]^{\mathrm{T}}+\left[\begin{array}{ll}
\hat{\psi}_{1} & \hat{\psi}_{2}
\end{array}\right]\left[\begin{array}{ll}
z_{1} & z_{10}
\end{array}\right]^{\mathrm{T}} \\
& \hat{\Xi}_{2}=\left[\begin{array}{ll}
z_{1} & z_{10}
\end{array}\right]\left(\left(I \otimes \hat{R}_{2}\right)+\left(I \otimes \hat{R}_{3}\right)\right)\left[\begin{array}{ll}
z_{1} & z_{10}
\end{array}\right]^{\mathrm{T}} \\
& +\left[\begin{array}{ll}
z_{2} & z_{11}
\end{array}\right]\left(\left(I \otimes \hat{R}_{4}\right)-\left(I \otimes \hat{R}_{2}\right)\right)\left[\begin{array}{ll}
z_{2} & z_{11}
\end{array}\right]^{\mathrm{T}} \\
& -(1-\lambda)\left[\begin{array}{ll}
z_{3} & z_{12}
\end{array}\right]\left(I \otimes \hat{R}_{3}\right)\left[\begin{array}{ll}
z_{3} & z_{12}
\end{array}\right]^{\mathrm{T}} \\
& -\left[\begin{array}{ll}
z_{4} & z_{13}
\end{array}\right]\left(I \otimes \hat{R}_{4}\right)\left[\begin{array}{ll}
z_{3} & z_{13}
\end{array}\right]^{\mathrm{T}} \\
& \hat{\Xi}_{3}=\hat{\Xi}_{31}+\hat{\Xi}_{32}+\hat{\Xi}_{33}+\hat{\Xi}_{34}, \quad \hat{\Xi}_{31}=-\frac{1}{\varepsilon_{1}} \eta_{1} N \hat{\bar{R}}_{5} N^{\mathrm{T}} \eta_{1}^{\mathrm{T}} \\
& \hat{\Xi}_{32}=-\frac{1}{\varepsilon_{1}} \eta_{2} N \hat{\bar{R}}_{5} N^{\mathrm{T}} \eta_{2}^{\mathrm{T}}, \quad \hat{\Xi}_{33}=-\frac{1}{\varepsilon_{2}-\varepsilon_{1}} \eta_{3} N \hat{\bar{R}}_{6} N^{\mathrm{T}} \eta_{3}^{\mathrm{T}} \\
& \hat{\Xi}_{34}=-\frac{1}{\varepsilon_{2}-\varepsilon_{1}} \eta_{4} N \hat{\bar{R}}_{6} N^{\mathrm{T}} \eta_{4}^{\mathrm{T}} \\
& \hat{\Xi}_{4}=\left[\begin{array}{ll}
\Xi_{41} & \Xi_{42}
\end{array}\right]\left(I \otimes \hat{Q}_{1}\right)\left[z_{2}-z_{3} \quad z_{11}-z_{12}\right]^{\mathrm{T}} \\
& +\left[\begin{array}{ll}
\Xi_{41} & \Xi_{42}
\end{array}\right]\left(I \otimes \hat{Q}_{2}\right)\left[\begin{array}{ll}
z_{3}-z_{4} & z_{12}-z_{13}
\end{array}\right]^{\mathrm{T}} \\
& \hat{\Xi}_{5}=\Xi_{5} \text {. }
\end{aligned}
$$

Theorem 1: Let $(A, B)$ be stabilizable and $(A, C)$ be detectable. For prescribed scalars $\varepsilon_{1}>0, \varepsilon_{2}>0\left(\varepsilon_{2} \geq \varepsilon_{1}\right)$, $\lambda>0$, and $\vartheta_{k} \geq 0(k=1,2, \ldots, m)$, any appropriate dimensioned matrices $W_{a}(a=1,2)$, and known gain matrices $D$ and $K$, the leader-following consensus problem of the MAS (1) is asymptotically solved in the mean-square sense via the protocol (4) if there exist real constant matrices $R_{b}>0$ $(b=1,2, \ldots, 7)$ and any appropriate dimensioned matrices $Q_{a}(a=1,2)$ such that the following condition holds:

$$
\left[\begin{array}{cccc}
\Theta_{1} & \Pi_{1} & \Pi_{2} & \Pi_{3} \\
* & \Pi_{4} & 0 & 0 \\
* & * & \Pi_{5} & 0 \\
* & * & * & \Pi_{6}
\end{array}\right]<0
$$

where

$$
\begin{aligned}
& \Theta_{1}=\sum_{i=1}^{5} \Xi_{i}, \quad \psi_{3}=\left[\begin{array}{ll}
z_{3} & z_{12}
\end{array}\right] \\
& \psi_{4}=\left[\vartheta_{1} \psi_{3}\left(I \otimes B E_{1} K\right)^{\mathrm{T}} \quad \vartheta_{2} \psi_{3}\left(I \otimes B E_{2} K\right)^{\mathrm{T}} \quad \cdots\right. \\
& \left.\cdots \vartheta_{m} \psi_{3}\left(I \otimes B E_{m} K\right)^{\mathrm{T}}\right] \\
& \Pi_{1}=\left[z_{1}\left(I \otimes W_{1}\right) \quad z_{10}\left(I \otimes W_{2}\right)\right] \\
& \Pi_{2}=\left[\begin{array}{lll}
\Pi_{21} & \Pi_{22} & \Pi_{23}
\end{array}\right] \\
& \Pi_{21}=\left[\begin{array}{lll}
\sqrt{\varepsilon_{1}} \psi_{1}, & \sqrt{\varepsilon_{2}-\varepsilon_{1}} \psi_{1} & \sqrt{\varepsilon_{2}-\varepsilon_{1}} \psi_{1}
\end{array}\right] \\
& \Pi_{22}=\left[\begin{array}{lll}
\sqrt{\varepsilon_{1}} \psi_{2} & \sqrt{\varepsilon_{2}-\varepsilon_{1}} \psi_{2} & \sqrt{\varepsilon_{2}-\varepsilon_{1}} \psi_{2}
\end{array}\right] \\
& \Pi_{23}=\left[\begin{array}{lll}
\sqrt{\varepsilon_{1}} \psi_{4} & \sqrt{\varepsilon_{2}-\varepsilon_{1}} \psi_{4} & \sqrt{\varepsilon_{2}-\varepsilon_{1}} \psi_{4}
\end{array}\right] \\
& \Pi_{3}=\sqrt{\varepsilon_{2}-\varepsilon_{1}}\left[\begin{array}{ll}
\Pi_{31} & \Pi_{32}
\end{array}\right] \\
& \Pi_{31}=\left[\Xi_{41}\left(I \otimes Q_{1}\right) \quad \Xi_{42}\left(I \otimes Q_{1}\right)\right] \\
& \Pi_{32}=\left[\Xi_{41}\left(I \otimes Q_{2}\right) \quad \Xi_{42}\left(I \otimes Q_{2}\right)\right] \\
& \Pi_{4}=\operatorname{diag}\{-(I \otimes I),-(I \otimes I)\} \\
& \Pi_{5}=\operatorname{diag}\left\{-\Omega_{1},-\Omega_{1},-\Omega_{2}\right\} \\
& \Omega_{1}=\operatorname{diag}\left\{\left(I \otimes R_{5}\right),\left(I \otimes R_{6}\right),\left(I \otimes R_{7}\right)\right\} \\
& \Omega_{2}=\operatorname{diag}\left\{\Omega_{21}, \Omega_{22}, \Omega_{23}\right\}
\end{aligned}
$$




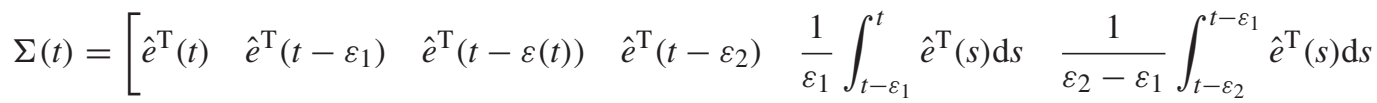

$$
\begin{aligned}
& \frac{2}{\varepsilon_{1}^{2}} \int_{t-\varepsilon_{1}}^{t} \int_{u}^{t} \hat{e}^{\mathrm{T}}(s) \mathrm{d} s \mathrm{~d} u \quad \frac{2}{\left(\varepsilon_{2}-\varepsilon_{1}\right)^{2}} \int_{t-\varepsilon_{2}}^{t-\varepsilon_{1}} \int_{u}^{t} \hat{e}^{\mathrm{T}}(s) \mathrm{d} s \mathrm{~d} u \quad G_{1}^{\mathrm{T}}(\hat{e}(t), t) \\
& e^{\mathrm{T}}(t) \quad e^{\mathrm{T}}\left(t-\varepsilon_{1}\right) \quad e^{\mathrm{T}}(t-\varepsilon(t)) \quad e^{\mathrm{T}}\left(t-\varepsilon_{2}\right) \quad \frac{1}{\varepsilon_{1}} \int_{t-\varepsilon_{1}}^{t} e^{\mathrm{T}}(s) \mathrm{d} s \quad \frac{1}{\varepsilon_{2}-\varepsilon_{1}} \int_{t-\varepsilon_{2}}^{t-\varepsilon_{1}} e^{\mathrm{T}}(s) \mathrm{d} s \\
& \left.\frac{2}{\varepsilon_{1}^{2}} \int_{t-\varepsilon_{1}}^{t} \int_{u}^{t} e^{\mathrm{T}}(s) \mathrm{d} s \mathrm{~d} u \quad \frac{2}{\left(\varepsilon_{2}-\varepsilon_{1}\right)^{2}} \int_{t-\varepsilon_{2}}^{t-\varepsilon_{1}} \int_{u}^{t} e^{\mathrm{T}}(s) \mathrm{d} s \mathrm{~d} u \quad G_{2}^{\mathrm{T}}(e(t), t)\right]^{\mathrm{T}}
\end{aligned}
$$

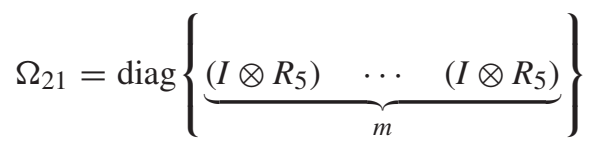

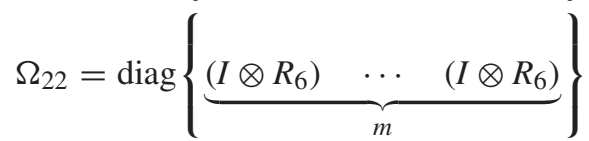

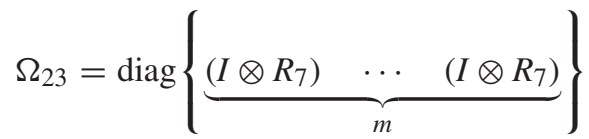

$$
\begin{aligned}
& \Pi_{6}=\operatorname{diag}\{\underbrace{-\left(I \otimes R_{7}\right), \ldots,-\left(I \otimes R_{7}\right)}_{4}\} . \\
& V(t)=V_{1}(t)+V_{2}(t)+V_{3}(t) \\
& +e^{\mathrm{T}}(t)\left[\left(I \otimes R_{2}\right)+\left(I \otimes R_{3}\right)\right] e(t) \\
& +e^{\mathrm{T}}\left(t-\varepsilon_{1}\right)\left[\left(I \otimes R_{4}\right)-\left(I \otimes R_{2}\right)\right] e\left(t-\varepsilon_{1}\right) \\
& -(1-\dot{\varepsilon}(t)) e^{\mathrm{T}}(t-\varepsilon(t))\left(I \otimes R_{3}\right) e(t-\varepsilon(t)) \\
& \left.-e^{\mathrm{T}}\left(t-\varepsilon_{2}\right)\left(I \otimes R_{4}\right) e\left(t-\varepsilon_{2}\right)\right\} \\
& \leq \mathbb{E}\left\{\Sigma^{\mathrm{T}}(t) \Xi_{2} \Sigma(t)\right\} \\
& \mathbb{E}\left\{\Delta V_{3}(t)\right\}=\mathbb{E}\left\{\dot { \hat { e } } ^ { \mathrm { T } } ( t ) \left[\varepsilon_{1}\left(I \otimes R_{5}\right)+\left(\varepsilon_{2}-\varepsilon_{1}\right)\left[\left(I \otimes R_{6}\right)\right.\right.\right. \\
& \left.\left.+\left(I \otimes R_{7}\right)\right]\right] \dot{\hat{e}}(t) \\
& -\int_{t-\varepsilon_{1}}^{t} \dot{\hat{e}}^{\mathrm{T}}(s)\left(I \otimes R_{5}\right) \dot{\hat{e}}(s) \mathrm{d} s \\
& -\int_{t-\varepsilon_{2}}^{t-\varepsilon_{1}} \dot{\hat{e}}^{\mathrm{T}}(s)\left[\left(I \otimes R_{6}\right)+\left(I \otimes R_{7}\right)\right] \dot{\hat{e}}(s) \mathrm{d} s \\
& +\dot{e}^{\mathrm{T}}(t)\left[\varepsilon_{1}\left(I \otimes R_{5}\right)+\left(\varepsilon_{2}-\varepsilon_{1}\right)\left[\left(I \otimes R_{6}\right)\right.\right. \\
& \left.\left.+\left(I \otimes R_{7}\right)\right]\right] \dot{e}(t) \\
& -\int_{t-\varepsilon_{1}}^{t} \dot{e}^{\mathrm{T}}(s)\left(I \otimes R_{5}\right) \dot{e}(s) \mathrm{d} s \\
& \left.-\int_{t-\varepsilon_{2}}^{t-\varepsilon_{1}} \dot{e}^{\mathrm{T}}(s)\left[\left(I \otimes R_{6}\right)+\left(I \otimes R_{7}\right)\right] \dot{e}(s) \mathrm{d} s\right\} .
\end{aligned}
$$

where

$$
\begin{aligned}
V_{1}(t)= & \Lambda^{\mathrm{T}}(t) \mathcal{R}_{1} \Lambda(t) \\
V_{2}(t)= & \int_{t-\varepsilon_{1}}^{t} \Lambda^{\mathrm{T}}(s) \mathcal{R}_{2} \Lambda(s) \mathrm{d} s+\int_{t-\varepsilon(t)}^{t} \Lambda^{\mathrm{T}}(s) \mathcal{R}_{3} \Lambda(s) \mathrm{d} s \\
& +\int_{t-\varepsilon_{2}}^{t-\varepsilon_{1}} \Lambda^{\mathrm{T}}(s) \mathcal{R}_{4} \Lambda(s) \mathrm{d} s \\
V_{3}(t)= & \int_{-\varepsilon_{1}}^{0} \int_{t+u}^{t} \Lambda^{\mathrm{T}}(s) \mathcal{R}_{5} \Lambda(s) \mathrm{d} s \mathrm{~d} u \\
& +\int_{-\varepsilon_{2}}^{-\varepsilon_{1}} \int_{t+u}^{t} \Lambda^{\mathrm{T}}(s)\left[\mathcal{R}_{6}+\mathcal{R}_{7}\right] \Lambda(s) \mathrm{d} s \mathrm{~d} u
\end{aligned}
$$

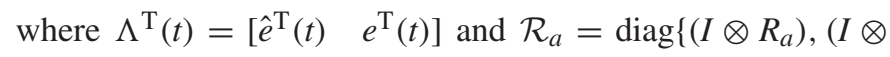
$\left.\left.R_{a}\right)\right\}(a=1,2, \ldots, 7)$.

Then, by using the infinitesimal operator in (9) and taking the mathematical expectation, it is easy to find that

$$
\begin{aligned}
\mathbb{E}\left\{\Delta V_{1}(t)\right\}=\mathbb{E}\{ & \hat{e}^{\mathrm{T}}(t)\left(I \otimes R_{1}\right) \dot{\hat{e}}(t)+\dot{\hat{e}}^{\mathrm{T}}(t)\left(I \otimes R_{1}\right) \hat{e}(t) \\
& \left.+e^{\mathrm{T}}(t)\left(I \otimes R_{1}\right) \dot{e}(t)+\dot{e}^{\mathrm{T}}(t)\left(I \otimes R_{1}\right) e(t)\right\} \\
=\mathbb{E}\{ & \left\{\Sigma^{\mathrm{T}}(t) \Xi_{1} \Sigma(t)\right\} \\
\mathbb{E}\left\{\Delta V_{2}(t)\right\}=\mathbb{E}\{ & \hat{e}^{\mathrm{T}}(t)\left[\left(I \otimes R_{2}\right)+\left(I \otimes R_{3}\right)\right] \hat{e}(t) \\
& +\hat{e}^{\mathrm{T}}\left(t-\varepsilon_{1}\right)\left[\left(I \otimes R_{4}\right)-\left(I \otimes R_{2}\right)\right] \hat{e}\left(t-\varepsilon_{1}\right) \\
& -(1-\dot{\varepsilon}(t)) \hat{e}^{\mathrm{T}}(t-\varepsilon(t))\left(I \otimes R_{3}\right) \hat{e}(t-\varepsilon(t)) \\
& -\hat{e}^{\mathrm{T}}\left(t-\varepsilon_{2}\right)\left(I \otimes R_{4}\right) \hat{e}\left(t-\varepsilon_{2}\right)
\end{aligned}
$$

Now, by applying the recently developed integral inequality named the "refined Jensen's inequality" [49] to the above integral terms containing $\left(I \otimes R_{5}\right)$ and $\left(I \otimes R_{6}\right)$, the following inequalities are obtained:

$$
\begin{aligned}
& -\int_{t-\varepsilon_{1}}^{t} \dot{\hat{e}}^{\mathrm{T}}(s)\left(I \otimes R_{5}\right) \dot{\hat{e}}(s) \mathrm{d} s \\
& \leq-\frac{1}{\varepsilon_{1}} \Sigma^{\mathrm{T}}(t) \eta_{1} N \bar{R}_{5} N^{\mathrm{T}} \eta_{1}^{\mathrm{T}} \Sigma(t)=\Sigma^{\mathrm{T}}(t) \Xi_{31} \Sigma(t) \\
& -\int_{t-\varepsilon_{1}}^{t} \dot{e}^{\mathrm{T}}(s)\left(I \otimes R_{5}\right) \dot{e}(s) \mathrm{d} s \\
& \leq-\frac{1}{\varepsilon_{1}} \Sigma^{\mathrm{T}}(t) \eta_{2} N \bar{R}_{5} N^{\mathrm{T}} \eta_{2}^{\mathrm{T}} \Sigma(t)=\Sigma^{\mathrm{T}}(t) \Xi_{32} \Sigma(t) \\
& -\int_{t-\varepsilon_{2}}^{t-\varepsilon_{1}} \frac{\dot{\hat{e}}^{\mathrm{T}}(s)\left(I \otimes R_{6}\right) \dot{\hat{e}}(s) \mathrm{d} s}{\leq-\frac{1}{\varepsilon_{2}-\varepsilon_{1}} \Sigma^{\mathrm{T}}(t) \eta_{3} N \bar{R}_{6} N^{\mathrm{T}} \eta_{3}^{\mathrm{T}} \Sigma(t)=\Sigma^{\mathrm{T}}(t) \Xi_{33} \Sigma(t)} \\
& -\int_{t-\varepsilon_{2}}^{t-\varepsilon_{1}} \dot{\hat{e}}^{\mathrm{T}}(s)\left(I \otimes R_{6}\right) \dot{\hat{e}}(s) \mathrm{d} s \\
& \leq-\frac{1}{\varepsilon_{2}-\varepsilon_{1}} \Sigma^{\mathrm{T}}(t) \eta_{4} N \bar{R}_{6} N^{\mathrm{T}} \eta_{4}^{\mathrm{T}} \Sigma(t)=\Sigma^{\mathrm{T}}(t) \Xi_{34} \Sigma(t) .
\end{aligned}
$$


Based on the definition of $\varepsilon(t)$, the integral terms containing $\left(I \otimes R_{7}\right)$ can be split as follows:

$$
\begin{aligned}
& \int_{t-\varepsilon_{2}}^{t-\varepsilon_{1}} \dot{\hat{e}}{ }^{\mathrm{T}}(s)\left(I \otimes R_{7}\right) \dot{\hat{e}}(s) \mathrm{d} s=\int_{t-\varepsilon_{2}}^{t-\varepsilon(t)} \dot{\hat{e}}^{\mathrm{T}}(s)\left(I \otimes R_{7}\right) \dot{\hat{e}}(s) \mathrm{d} s \\
& +\int_{t-\varepsilon(t)}^{t-\varepsilon_{1}} \dot{\hat{e}}^{\mathrm{T}}(s)\left(I \otimes R_{7}\right) \dot{\hat{e}}(s) \mathrm{d} s \\
& \int_{t-\varepsilon_{2}}^{t-\varepsilon_{1}} \dot{e}^{\mathrm{T}}(s)\left(I \otimes R_{7}\right) \dot{e}(s) \mathrm{d} s=\int_{t-\varepsilon_{2}}^{t-\varepsilon(t)} \dot{e}^{\mathrm{T}}(s)\left(I \otimes R_{7}\right) \dot{e}(s) \mathrm{d} s \\
& +\int_{t-\varepsilon(t)}^{t-\varepsilon_{1}} \dot{e}^{\mathrm{T}}(s)\left(I \otimes R_{7}\right) \dot{e}(s) \mathrm{d} s .
\end{aligned}
$$

Furthermore, for any appropriate dimensioned real constant

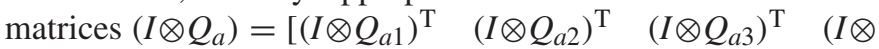
$\left.\left.Q_{a 4}\right)^{\mathrm{T}}\right]^{\mathrm{T}}(a=1,2)$, the following zero equations hold well:

$$
\begin{aligned}
& 2 \alpha^{\mathrm{T}}(t)\left(I \otimes Q_{1}\right) \\
& \times\left[\hat{e}\left(t-\varepsilon_{1}\right)-\hat{e}(t-\varepsilon(t))-\int_{t-\varepsilon(t)}^{t-\varepsilon_{1}} \dot{\hat{e}}(s) \mathrm{d} s\right]=0 \\
& 2 \beta^{\mathrm{T}}(t)\left(I \otimes Q_{1}\right) \\
& \times\left[e\left(t-\varepsilon_{1}\right)-e(t-\varepsilon(t))-\int_{t-\varepsilon(t)}^{t-\varepsilon_{1}} \dot{e}(s) \mathrm{d} s\right]=0 \\
& 2 \alpha^{\mathrm{T}}(t)\left(I \otimes Q_{2}\right) \\
& \times\left[\hat{e}(t-\varepsilon(t))-\hat{e}\left(t-\varepsilon_{2}\right)-\int_{t-\varepsilon_{2}}^{t-\varepsilon(t)} \dot{\hat{e}}(s) \mathrm{d} s\right]=0 \\
& 2 \beta^{\mathrm{T}}(t)\left(I \otimes Q_{2}\right) \\
& \times\left[e(t-\varepsilon(t))-e\left(t-\varepsilon_{2}\right)-\int_{t-\varepsilon_{2}}^{t-\varepsilon(t)} \dot{e}(s) \mathrm{d} s\right]=0 \\
& \left(\varepsilon(t)-\varepsilon_{1}\right) \alpha^{\mathrm{T}}(t) \varrho_{1} \alpha(t)-\int_{t-\varepsilon(t)}^{t-\varepsilon_{1}} \alpha^{\mathrm{T}}(t) \varrho_{1} \alpha(t) \mathrm{d} s=0 \\
& \left(\varepsilon(t)-\varepsilon_{1}\right) \beta^{\mathrm{T}}(t) \varrho_{1} \beta(t)-\int_{t-\varepsilon(t)}^{t-\varepsilon_{1}} \beta^{\mathrm{T}}(t) \varrho_{1} \beta(t) \mathrm{d} s=0 \\
& \left(\varepsilon_{2}-\varepsilon(t)\right) \alpha^{\mathrm{T}}(t) \varrho_{2} \alpha(t)-\int_{t-\varepsilon_{2}}^{t-\varepsilon(t)} \alpha^{\mathrm{T}}(t) \varrho_{2} \alpha(t) \mathrm{d} s=0 \\
& \left(\varepsilon_{2}-\varepsilon(t)\right) \beta^{\mathrm{T}}(t) \varrho_{2} \beta(t)-\int_{t-\varepsilon_{2}}^{t-\varepsilon(t)} \beta^{\mathrm{T}}(t) \varrho_{2} \beta(t) \mathrm{d} s=0
\end{aligned}
$$

where $\alpha(t)=\left[\begin{array}{llll}\hat{e}^{\mathrm{T}}(t) & \hat{e}^{\mathrm{T}}(t-\varepsilon(t)) & e^{\mathrm{T}}(t) & e^{\mathrm{T}}(t-\varepsilon(t))\end{array}\right]^{\mathrm{T}}$, $\beta(t)=\left[\begin{array}{llll}\hat{e}^{\mathrm{T}}\left(t-\varepsilon_{1}\right) & \hat{e}^{\mathrm{T}}\left(t-\varepsilon_{2}\right) & e^{\mathrm{T}}\left(t-\varepsilon_{1}\right) & e^{\mathrm{T}}\left(t-\varepsilon_{2}\right)\end{array}\right]^{\mathrm{T}}$, $\varrho_{1}=\left(I \otimes Q_{1}\right)\left(I \otimes R_{7}\right)^{-1}\left(I \otimes Q_{1}\right)^{\mathrm{T}}$, and $\varrho_{2}=\left(I \otimes Q_{2}\right)(I \otimes$ $\left.R_{7}\right)^{-1}\left(I \otimes Q_{2}\right)^{\mathrm{T}}$.

By denoting $\Omega=\varepsilon_{1}\left(I \otimes R_{5}\right)+\left(\varepsilon_{2}-\varepsilon_{1}\right)\left[\left(I \otimes R_{6}\right)+\left(I \otimes R_{7}\right)\right]$ and using the facts $\mathbb{E}\{H\}=\bar{H}, \mathbb{E}\{H-\bar{H}\}=0$, and $\mathbb{E}\{(H-$ $\bar{H}) \Omega(H-\bar{H})\}=\sum_{k=1}^{m} \vartheta_{k}^{2} E_{k}^{\mathrm{T}} \Omega E_{k}$ in $\mathbb{E}\left\{\Delta V_{3}(t)\right\}$, we can obtain

$$
\begin{aligned}
\mathbb{E}\left\{\dot{\hat{e}}^{\mathrm{T}}(t) \Omega \dot{\hat{e}}(t)\right\}=\mathbb{E}\left\{\Sigma^{\mathrm{T}}(t) \psi_{1} \Omega \psi_{1}^{\mathrm{T}} \Sigma(t)\right\} \\
\mathbb{E}\left\{\dot{e}^{\mathrm{T}}(t) \Omega \dot{e}(t)\right\}=\mathbb{E}\left\{\Sigma^{\mathrm{T}}(t) \psi_{2} \Omega \psi_{2}^{\mathrm{T}} \Sigma(t)\right. \\
+\hat{e}^{\mathrm{T}}(t-\varepsilon(t)) \Upsilon \hat{e}(t-\varepsilon(t)) \\
\left.+e^{\mathrm{T}}(t-\varepsilon(t)) \Upsilon e(t-\varepsilon(t))\right\}
\end{aligned}
$$

where $\Upsilon=\sum_{k=1}^{m} \vartheta_{k}^{2}\left(I \otimes B E_{k} K\right)^{\mathrm{T}} \Omega\left(I \otimes B E_{k} K\right)$. Based on our assumption for the nonlinear term, we gain the following inequalities:

$$
\begin{aligned}
& 0 \leq \hat{e}^{\mathrm{T}}(t)\left(I \otimes W_{1}\right)^{\mathrm{T}}\left(I \otimes W_{1}\right) \hat{e}(t)-G_{1}^{\mathrm{T}}(\hat{e}(t), t) G_{1}(\hat{e}(t), t) \\
& 0 \leq e^{\mathrm{T}}(t)\left(I \otimes W_{2}\right)^{\mathrm{T}}\left(I \otimes W_{2}\right) e(t)-G_{2}^{\mathrm{T}}(e(t), t) G_{2}(e(t), t) .
\end{aligned}
$$

By collecting all the equations and inequalities involved in the estimation of $\mathbb{E}\{\Delta V(t)\}$, it is easy to obtain $\mathbb{E}\{\Delta V(t)\} \leq$ $\mathbb{E}\left\{\Sigma^{\mathrm{T}}(t) \Theta \Sigma(t)\right\}$, where

$$
\begin{aligned}
\Theta= & \sum_{a=1}^{5} \Xi_{a}+\psi_{1} \Omega \psi_{1}^{\mathrm{T}}+\psi_{2} \Omega \psi_{2}^{\mathrm{T}}+\left[\begin{array}{ll}
z_{1} & z_{10}
\end{array}\right] \\
& \times \operatorname{diag}\left\{\left(I \otimes W_{1}\right)\left(I \otimes W_{1}\right)^{\mathrm{T}},\left(I \otimes W_{2}\right)\left(I \otimes W_{2}\right)^{\mathrm{T}}\right\} \\
& \times\left[\begin{array}{ll}
z_{1} & z_{10}
\end{array}\right]^{\mathrm{T}}+\left[\begin{array}{ll}
z_{3} & z_{12}
\end{array}\right] \Upsilon\left[\begin{array}{ll}
z_{3} & z_{12}
\end{array}\right]^{\mathrm{T}} \\
& +\left(\varepsilon_{2}-\varepsilon_{1}\right) \Gamma\left(I \otimes R_{7}\right)^{-1} \Gamma^{\mathrm{T}} \\
\Gamma= & {\left[\Xi_{41}\left(I \otimes Q_{1}\right) \Xi_{42}\left(I \otimes Q_{1}\right) \Xi_{41}\left(I \otimes Q_{2}\right) \Xi_{42}\left(I \otimes Q_{2}\right)\right] . }
\end{aligned}
$$

Now, by using the Schur complement [34] in $\Theta$, it seems easy to find that $\Theta$ is an equivalent form of the left-hand side of (8). Then, it is clear that $\mathbb{E}\{\Delta V(t)\}<0$ when the inequality (8) holds and the observer and reliable control gain matrices are known. Thus, based on Lyapunov-Krasovskii stability theory, the closed-loop error systems (6) and (7) are mean-square asymptotically stable, which means that the considered MAS (1) can achieve the leader-following consensus via the reliable control (4).

In the following theorem, based on the condition obtained in Theorem 1, we will develop a consensus protocol for the observer-based and stochastic reliable state feedback controllers that guarantees the leader-following consensus of the MAS (1) even in the case of unknown state feedback control gains.

Theorem 2: Consider the MAS (1) with the assumptions that $(A, B)$ and $(A, C)$ are stabilizable and detectable, respectively. Let $\varepsilon_{1}>0, \varepsilon_{2}>0\left(\varepsilon_{2} \geq \varepsilon_{1}\right), \lambda>0, \rho>0$, and $\vartheta_{k} \geq 0$ $(k=1,2, \ldots, m)$ be given scalars and $W_{a}(a=1,2)$ be any matrices with appropriate dimensions. If there exist real constant matrices $\hat{R}_{b}>0(b=1,2, \ldots, 7)$ and any real constant matrices $\hat{Q}_{a}(a=1,2)$ with appropriate dimensions satisfying the following LMI:

$$
\left[\begin{array}{cccc}
\hat{\Theta}_{1} & \hat{\Pi}_{1} & \hat{\Pi}_{2} & \hat{\Pi}_{3} \\
* & \hat{\Pi}_{4} & 0 & 0 \\
* & * & \hat{\Pi}_{5} & 0 \\
* & * & * & \hat{\Pi}_{6}
\end{array}\right]<0
$$

where

$$
\begin{aligned}
& \hat{\Theta}_{1}=\sum_{a=1}^{5} \hat{\Xi}_{a}, \quad \psi_{3}=\left[\begin{array}{ll}
z_{3} & z_{12}
\end{array}\right] \\
& \hat{\psi}_{4}=\left[\vartheta_{1} \psi_{3}\left(I \otimes B E_{1} Y_{2}\right)^{\mathrm{T}} \quad \vartheta_{2} \psi_{3}\left(I \otimes B E_{2} Y_{2}\right)^{\mathrm{T}} \quad \cdots\right. \\
& \left.\vartheta_{m} \psi_{3}\left(I \otimes B E_{m} Y_{2}\right)^{\mathrm{T}}\right] \\
& \Pi_{1}=\left[z_{1}\left(I \otimes P_{1}\right)\left(I \otimes W_{1}\right) \quad z_{10}\left(I \otimes P_{1}\right)\left(I \otimes W_{2}\right)\right] \\
& \hat{\Pi}_{2}=\left[\begin{array}{lll}
\hat{\Pi}_{21} & \hat{\Pi}_{22} & \hat{\Pi}_{23}
\end{array}\right] \\
& \hat{\Pi}_{21}=\left[\begin{array}{lll}
\sqrt{\varepsilon_{1}} \hat{\psi}_{1} & \sqrt{\varepsilon_{2}-\varepsilon_{1}} \hat{\psi}_{1} & \sqrt{\varepsilon_{2}-\varepsilon_{1}} \hat{\psi}_{1}
\end{array}\right] \\
& \hat{\Pi}_{22}=\left[\begin{array}{lll}
\sqrt{\varepsilon_{1}} \hat{\psi}_{2} & \sqrt{\varepsilon_{2}-\varepsilon_{1}} \hat{\psi}_{2} & \sqrt{\varepsilon_{2}-\varepsilon_{1}} \hat{\psi}_{2}
\end{array}\right]
\end{aligned}
$$




$$
\begin{aligned}
& \hat{\Pi}_{23}=\left[\begin{array}{lll}
\sqrt{\varepsilon_{1}} \hat{\psi}_{4} & \sqrt{\varepsilon_{2}-\varepsilon_{1}} \hat{\psi}_{4} & \sqrt{\varepsilon_{2}-\varepsilon_{1}} \hat{\psi}_{4}
\end{array}\right] \\
& \hat{\Pi}_{3}=\sqrt{\varepsilon_{2}-\varepsilon_{1}}\left[\begin{array}{ll}
\hat{\Pi}_{31} & \hat{\Pi}_{32}
\end{array}\right] \\
& \hat{\Pi}_{31}=\left[\Xi_{41}\left(I \otimes \hat{Q}_{1}\right) \quad \Xi_{42}\left(I \otimes \hat{Q}_{1}\right)\right] \\
& \hat{\Pi}_{32}=\left[\Xi_{41}\left(I \otimes \hat{Q}_{2}\right) \quad \Xi_{42}\left(I \otimes \hat{Q}_{2}\right)\right] \\
& \hat{\Pi}_{4}=\operatorname{diag}\{-(I \otimes I),-(I \otimes I)\} \\
& \hat{\Pi}_{5}=\operatorname{diag}\left\{-\hat{\Omega}_{1},-\hat{\Omega}_{1},-\hat{\Omega}_{2}\right\} \\
& \Phi_{a}=-2 \rho\left(I \otimes P_{1}\right)+\rho^{2}\left(I \otimes \hat{R}_{a}\right)(a=5,6,7) \\
& \hat{\Omega}_{1}=\operatorname{diag}\left\{\Phi_{5}, \Phi_{6}, \Phi_{7}\right\}
\end{aligned}
$$

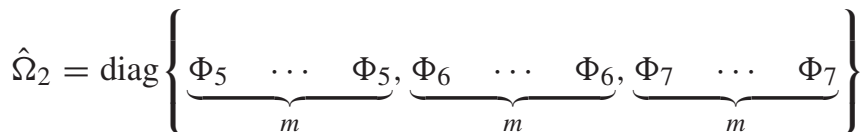

$$
\begin{aligned}
& \hat{\Pi}_{6}=\operatorname{diag}\{\underbrace{-\left(I \otimes \hat{R}_{7}\right), \ldots,-\left(I \otimes \hat{R}_{7}\right)}_{4}\} \\
& \hat{Q}_{a}=\left[\left(I \otimes \hat{Q}_{a 1}\right)^{\mathrm{T}}\left(I \otimes \hat{Q}_{a 2}\right)^{\mathrm{T}}\left(I \otimes \hat{Q}_{a 3}\right)^{\mathrm{T}}\left(I \otimes \hat{Q}_{a 4}\right)^{\mathrm{T}}\right]^{\mathrm{T}}
\end{aligned}
$$

$(a=1,2)$, then the MAS (1) achieves the leader-following consensus under the reliable protocol (4). Moreover, if the above LMI problem has a feasible solution, the observer and reliable state feedback control gain matrices can be computed from the following relations, respectively: $D=Y_{1} \hat{P}_{1}^{-1}$ and $K=Y_{2} P_{1}^{-1}$.

Proof: It is noted that based on the inequality (8), the MAS (1) under study asymptotically achieves the leaderfollowing consensus in the mean-square sense. Next, to develop a design for both the observer and state feedback controllers, we define the following notations: $P_{1}=R_{1}^{-1}$, $\hat{R}_{a}=P_{1} R_{a} P_{1} \quad(a=2,3, \ldots, 7)$, and $\hat{Q}_{a b}=P_{1} Q_{a b} P_{1}$ ( $a=1,2 ; b=1,2,3,4)$. Then, the left-hand side of (8) is pre and postmultiplied by $\Psi$ and its transpose, respectively, where

$$
\begin{aligned}
& \Psi=\operatorname{diag}\left\{\Psi_{1}, \Psi_{1},(I \otimes I), \Psi_{1}, \Psi_{1},(I \otimes I),(I \otimes I),(I \otimes I)\right. \\
& \left.\Psi_{2}, \Psi_{2}, \Psi_{3}, \Psi_{4}, \Psi_{5}, \Psi_{1}\right\} \\
& \Psi_{1}=\operatorname{diag}\{\underbrace{\left(I \otimes P_{1}\right), \ldots,\left(I \otimes P_{1}\right)}_{4}\} \\
& \Psi_{2}=\operatorname{diag}\left\{\left(I \otimes R_{5}\right)^{-1},\left(I \otimes R_{6}\right)^{-1},\left(I \otimes R_{7}\right)^{-1}\right\} \\
& \Psi_{3}=\operatorname{diag}\{\underbrace{\left(I \otimes R_{5}\right)^{-1}, \ldots,\left(I \otimes R_{5}\right)^{-1}}_{m}\} \\
& \Psi_{4}=\operatorname{diag}\{\underbrace{\left(I \otimes R_{6}\right)^{-1}, \ldots,\left(I \otimes R_{6}\right)^{-1}}_{m}\} \\
& \Psi_{5}=\operatorname{diag}\{\underbrace{\left(I \otimes R_{7}\right)^{-1}, \ldots,\left(I \otimes R_{7}\right)^{-1}}_{m}\} \text {. }
\end{aligned}
$$

Moreover, during the pre and postmultiplication, the term $C P_{1}$ can be equivalently written as $\hat{P}_{1} C$ with the help of Lemma 1 , where $\hat{P}_{1}=U S P_{11}^{-1} S^{-1} U^{-1}$, and by defining $Y_{1}=D \hat{P}_{1}$ and
$Y_{2}=K P_{1}$, the inequality (12) is obtained. Thus, the leaderfollowing consensus of the MAS (1) is attained under the reliable protocol (4).

Remark 2: The established consensus criterion in Theorems 1 and 2 is expressed in terms of LMIs, wherein the free-weighting matrix method is employed in the derivation of the main results to reduce possible conservatism. However, the introduction of free-weighting matrices will significantly increase the number of decision variables in the LMI-based consensus criterion. A large number of decision variables in an LMI make the consensus criterion more complex, since the computational burden is large and solving the LMI in question is very time-consuming. Fortunately, all the computations in the main results are off-line, and with the aid of the existing standard convex optimization software, the proposed LMI-based consensus criterion can be easily solved.

Remark 3: To date, the literature has included a few works concerned with the observer-based consensus analysis of various kinds of MASs [20], [31]-[33]. In all these works, the effect of actuator faults, which cannot be ignored when designing a robust controller [50], has not been considered. Thus, the main intention of this paper is to fill this gap by making the first attempt to deal with the problem of the observerbased leader-following consensus of nonlinear MASs under a stochastic faulty reliable controller. Therefore, the theoretical results proposed in this paper enrich the study of the observer-based consensus of MASs.

\section{VAlidation Through Simulations}

To verify the developed LMI-based conditions in the preceding section, here we present a numerical example with simulations. For convenience, let us take a nonlinear MAS with four agents and a leader, which can be modeled as (1), and the corresponding state coefficient matrices are given by

$$
\begin{aligned}
A & =\left[\begin{array}{cc}
-1.5 & 0.5 \\
0.8 & -0.3
\end{array}\right], B=\left[\begin{array}{l}
0.06 \\
0.01
\end{array}\right], C=\left[\begin{array}{ll}
0 & 1
\end{array}\right] \\
g\left(x_{i}(t), t\right) & =\left[\begin{array}{c}
0.01 \cos \left(x_{i 1}(t)\right) \\
0.01 \cos \left(x_{i 2}(t)\right)
\end{array}\right] .
\end{aligned}
$$

Clearly, the matrix pairs $(A, B)$ and $(A, C)$ are stabilizable and detectable, respectively. The interactions among the four agents and leader are described by three arbitrarily switched undirected topologies and depicted in Fig. 1, where the leader node is denoted by 0 and the following agents' nodes are denoted by 1,2,3,4. Moreover, in Fig. 1, the dashed lines show the interaction between the leader and the following agents, while the solid lines show the interactions among the neighboring agents. Based on these interactions, we can choose the following parameters:

$$
\begin{aligned}
L_{1} & =\left[\begin{array}{cccc}
2 & -1 & -1 & 0 \\
-1 & 2 & 0 & -1 \\
-1 & 0 & 2 & -1 \\
0 & -1 & -1 & 2
\end{array}\right] \\
L_{2} & =\left[\begin{array}{cccc}
2 & -1 & -1 & 0 \\
-1 & 2 & -1 & 0 \\
-1 & -1 & 3 & -1 \\
0 & 0 & -1 & 1
\end{array}\right]
\end{aligned}
$$



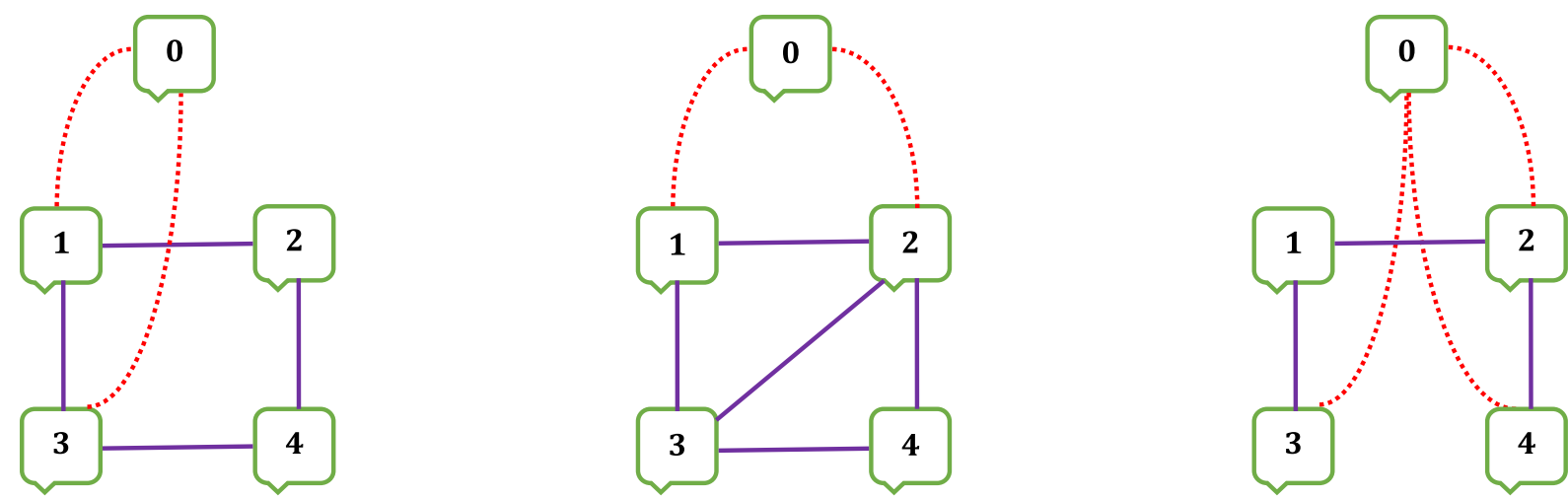

Fig. 1. Three different types of switching topologies for four agents and a leader.

$$
\begin{aligned}
L_{3} & =\left[\begin{array}{cccc}
2 & -1 & -1 & 0 \\
-1 & 2 & 0 & -1 \\
-1 & 0 & 1 & 0 \\
0 & -1 & 0 & 1
\end{array}\right], \quad F_{1}=\operatorname{diag}\{1,0,1,0\} \\
F_{2} & =\operatorname{diag}\{1,1,0,0\}, \quad F_{3}=\operatorname{diag}\{0,1,1,1\} .
\end{aligned}
$$

Furthermore, the time-varying delay $\varepsilon(t)$ is selected with the lower and upper bounds of $\varepsilon_{1}=0.3$ and $\varepsilon_{2}=11.5$, respectively. Additionally, its maximum time derivative limit is 0.4 . In this example, since $m=1$, the stochastic variable $\delta_{1}$ is assumed to obey Gaussian distribution with mean 0.2 and variance 0.5 .

Now, our intention is to design the reliable controller $u_{i}^{f}(t)$ such that the following agents are able to trace the leader's motion exactly even with the occurrence of probabilitydependent actuator faults and time-varying delay, or $x_{i}(t) \rightarrow$ $x_{0}(t)$ and $\hat{x}_{i}(t) \rightarrow x_{0}(t)$ for $i=1,2,3,4$. In this regard, the sufficient conditions obtained in Theorem 2 are employed to facilitate the control synthesis and attain the desired consensus of the MAS (1).

By using the values specified above and any standard numerical software to solve the LMI (12) with $\rho=40$, the observer and reliable state feedback control gain matrices are found to be

$$
D=\left[\begin{array}{c}
-0.0848 \\
0.4062
\end{array}\right], \quad K=\left[\begin{array}{ll}
0.0087 & 0.0190
\end{array}\right] .
$$

Subsequently, a simulation is conducted to show the potential of the designed consensus control law. By applying the above-estimated gain values to the considered MAS (1) with the initial conditions $x_{0}(0)=\left[\begin{array}{ll}3 & 1\end{array}\right]^{\mathrm{T}}, x_{1}(0)=$ $\left[\begin{array}{ll}13 & -4.5\end{array}\right]^{\mathrm{T}}, x_{2}(0)=\left[\begin{array}{ll}-1.5 & 3.5\end{array}\right]^{\mathrm{T}}, x_{3}(0)=\left[\begin{array}{ll}1 & 2.2\end{array}\right]^{\mathrm{T}}$, $x_{4}(0)=\left[\begin{array}{ll}-4.2 & 5\end{array}\right]^{\mathrm{T}}, \hat{x}_{1}(0)=\left[\begin{array}{ll}7 & 2\end{array}\right]^{\mathrm{T}}, \hat{x}_{2}(0)=\left[\begin{array}{ll}1.2 & 6.5\end{array}\right]^{\mathrm{T}}$, $\hat{x}_{3}(0)=\left[\begin{array}{ll}-4 & -2\end{array}\right]^{\mathrm{T}}$, and $\hat{x}_{4}(0)=\left[\begin{array}{ll}5 & -2\end{array}\right]^{\mathrm{T}}$, the trajectories of the actual state, its observer, and the leader are shown in Figs. 2-5. More precisely, the state trajectories of the first to fourth agents along with their observers and leader are displayed in Figs. 2-5, respectively. In these figures, it is clear that all four agents' states and their observers' trajectories are synchronized with the leader's trajectories, which means that the leader-following consensus of the nonlinear MAS (1) is attained in a very short time through the reliable control (4). Thus, the proposed control law (4) performs well even when including the effects of time-varying delay and

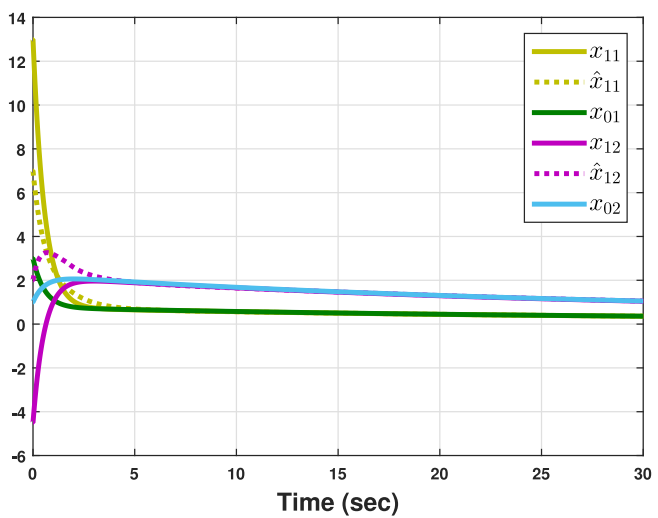

Fig. 2. State trajectories of first agent.

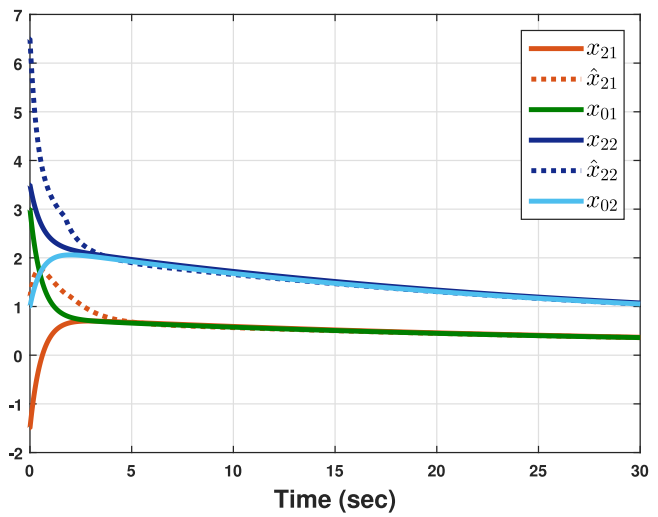

Fig. 3. State trajectories of second agent.

randomly occurring actuator faults. In addition, Fig. 6 gives the control response curves, and Fig. 7 depicts the region of attraction for different values of $\varepsilon_{2}$. It can be observed from Fig. 7 that the increment of the delay bound leads to a smaller region of attraction.

On the other hand, we estimate the maximum allowable upper bound (MAUB) of $\varepsilon_{2}$ for various values of $\varepsilon_{1}$ and $\lambda$, which is provided in Table I. From this table, it can be inferred that if we increase the lower bound $\varepsilon_{1}$ of time-varying delay $\varepsilon(t)$, the corresponding upper bound $\varepsilon_{2}$ also increases, and if we increase the derivative bound $\lambda$ of $\varepsilon(t)$, the corresponding upper bound $\varepsilon_{2}$ decreases. 
TABLE I

ESTIMATED MAUB OF $\varepsilon_{2}$ FOR DIFFERENT VALUES OF $\varepsilon_{1}$ AND $\lambda$

\begin{tabular}{|c||cccccc|}
\hline \multicolumn{1}{|c||}{$\varepsilon^{\varepsilon_{1}}$} & 0.4 & 0.5 & 0.6 & 0.7 & 0.8 & 0.9 \\
\hline \hline 0.4 & 13.8050 & 13.8605 & 13.9100 & 13.9612 & 14.0100 & 14.0590 \\
\hline 0.5 & 13.7732 & 13.8298 & 13.8801 & 13.9265 & 13.9814 & 14.0300 \\
\hline 0.6 & 13.7400 & 13.8021 & 13.8510 & 13.9030 & 13.9511 & 14.0020 \\
\hline 0.7 & 13.7142 & 13.7712 & 13.8202 & 13.8717 & 13.9220 & 13.9697 \\
\hline
\end{tabular}

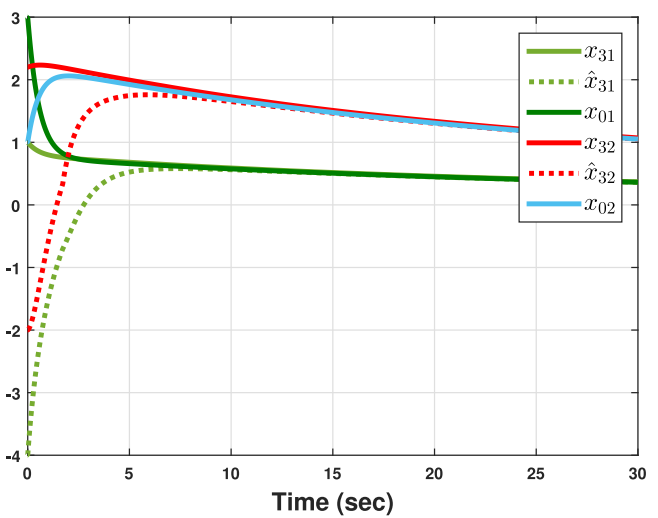

Fig. 4. State trajectories of third agent.

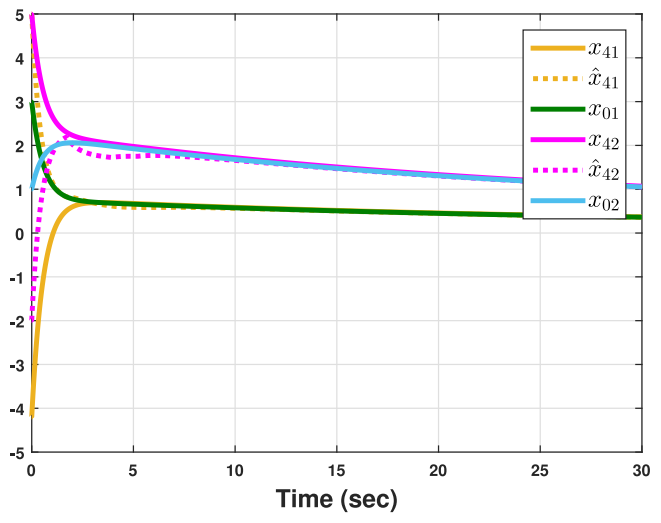

Fig. 5. State trajectories of fourth agent.

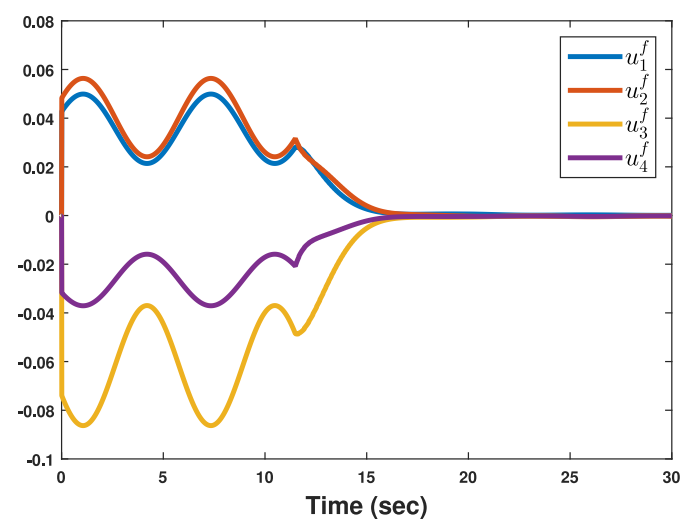

Fig. 6. Control responses.

As a result, it is found through the numerical simulations that both the trajectories of the actual state and its observer can follow the exact trajectories of the leader within a short

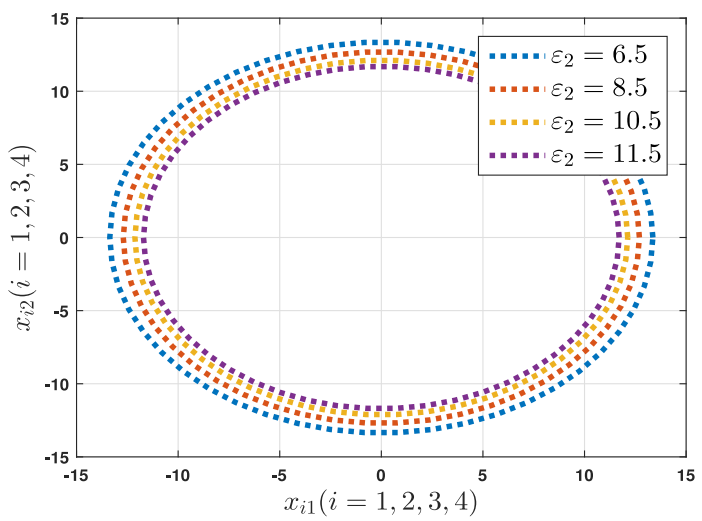

Fig. 7. Region of attraction.

period, which shows that the proposed observer-based protocol can accurately determine the immeasurable states of the nonlinear MAS (1). Furthermore, this example exhibits that the proposed method can be effectively employed in the verification of the leader-following consensus of the nonlinear MAS with the advantage that we can consider the stochastic actuator faults in the control strategy.

\section{CONCLUSion}

In this paper, by using the observer technique, the reliable control design problem has been examined for attaining the leader-following consensus of the nonlinear MAS subject to nondeterministic actuator faults and a switching topology. To be precise, an observer-based protocol and a probabilitydependent actuator fault model have been considered for the nonlinear MAS. Then, by defining simple linear transformations, two error systems have been formulated such that the leader-following consensus problem is equally changed to the stabilization problem. By properly choosing a delay-dependent Lyapunov-Krasovskii functional and utilizing some Kronecker product properties, the required conditions for ensuring the leader-following consensus of the nonlinear MAS have been obtained, whether actuator faults appear or not. Moreover, the design procedure of the reliable controller containing stochastic faults has been presented based on the obtained conditions. Lastly, the established theoretical results including the control design have been validated through a numerical example. It would be interesting to extend the proposed results to second-order MASs with communication delays using a nonfragile control scheme, which will be the topic of our future research. 


\section{REFERENCES}

[1] Y. Wu, H. Su, P. Shi, Z. Shu, and Z.-G. Wu, "Consensus of multiagent systems using aperiodic sampled-data control," IEEE Trans. Cybern., vol. 46, no. 9, pp. 2132-2143, Sep. 2016.

[2] Q. Shen and P. Shi, "Output consensus control of multiagent systems with unknown nonlinear dead zone," IEEE Trans. Syst., Man, Cybern., Syst., vol. 46, no. 10, pp. 1329-1337, Oct. 2016.

[3] H. Li, X. Liao, and T. Huang, "Second-order locally dynamical consensus of multiagent systems with arbitrarily fast switching directed topologies," IEEE Trans. Syst., Man, Cybern., Syst., vol. 43, no. 6, pp. 1343-1353, Nov. 2013.

[4] X. Qing, H. R. Karimi, Y. Niu, and X. Wang, "Decentralized unscented Kalman filter based on a consensus algorithm for multi-area dynamic state estimation in power systems," Int. J. Elect. Power Energy Syst., vol. 65, pp. 26-33, Feb. 2015.

[5] H. Li, Y. Shi, and W. Yan, "Distributed receding horizon control of constrained nonlinear vehicle formations with guaranteed $\gamma$-gain stability," Automatica, vol. 68, pp. 148-154, Jun. 2016.

[6] M. Rehan, A. Jameel, and C. K. Ahn, "Distributed consensus control of one-sided Lipschitz nonlinear multiagent systems," IEEE Trans. Syst., Man, Cybern., Syst., to be published, doi: 10.1109/TSMC.2017.2667701.

[7] H. Li, P. Xie, and W. Yan, "Receding horizon formation tracking control of constrained underactuated autonomous underwater vehicles," IEEE Trans. Ind. Electron., vol. 64, no. 6, pp. 5004-5013, Jun. 2017.

[8] Y. Zhao, B. Li, J. Qin, H. Gao, and H. R. Karimi, " $H_{\infty}$ consensus and synchronization of nonlinear systems based on a novel fuzzy model," IEEE Trans. Cybern., vol. 43, no. 6, pp. 2157-2169, Dec. 2013.

[9] S. Yang, Q. Liu, and J. Wang, "Distributed optimization based on a multiagent system in the presence of communication delays," IEEE Trans. Syst., Man, Cybern., Syst., vol. 47, no. 5, pp. 717-728, May 2017.

[10] Y. Tang, X. Xing, H. R. Karimi, L. Kocarev, and J. Kurths, "Tracking control of networked multi-agent systems under new characterizations of impulses and its applications in robotic systems," IEEE Trans. Ind. Electron., vol. 63, no. 2, pp. 1299-1307, Feb. 2016.

[11] W. Hou, M. Y. Fu, and H. Zhang, "Consensusability of linear multi-agent systems with time delay," Int. J. Robust Nonlin. Control, vol. 26 no. 12, pp. 2529-2541, Aug. 2016.

[12] J. Xi, Y. Yu, G. Liu, and Y. Zhong, "Guaranteed-cost consensus for singular multi-agent systems with switching topologies," IEEE Trans. Circuits Syst. I, Reg. Papers, vol. 61, no. 5, pp. 1531-1542, May 2014.

[13] W. Xing, Y. Zhao, and H. R. Karimi, "Convergence analysis on multi-AUV systems with leader-follower architecture," IEEE Access, vol. 5, pp. 853-868, 2017.

[14] H. Liu, H. R. Karimi, S. Du, W. Xia, and C. Zhong, "Leader-following consensus of discrete-time multiagent systems with time-varying delay based on large delay theory," Inf. Sci., vol. 417, pp. 236-246, Nov. 2017.

[15] J. Wang, Z. Duan, G. Wen, and G. Chen, "Leader-following consensus of networked second-order agents with delayed information transmission," IET Control Theory Appl., vol. 8, no. 14, pp. 1421-1428, Sep. 2014.

[16] H. Su, M. Z. Q. Chen, J. Lam, and Z. Lin, "Semi-global leader-following consensus of linear multi-agent systems with input saturation via low gain feedback," IEEE Trans. Circuits Syst. I, Reg. Papers, vol. 60, no. 7, pp. 1881-1889, Jul. 2013.

[17] W. Ni and X. Wang, "Leader-following consensus of high-order multi-agent systems with bounded transmission channels," Int. J. Syst. Sci., vol. 44, no. 9, pp. 1711-1725, Sep. 2013.

[18] Y. Zhao, Z. Li, and Z. Duan, "Distributed consensus tracking of multi-agent systems with nonlinear dynamics under a reference leader," Int. J. Control, vol. 86, no. 10, pp. 1859-1869, May 2013.

[19] Q. Song, F. Liu, J. Cao, and W. Yu, " $M$-matrix strategies for pinningcontrolled leader-following consensus in multiagent systems with nonlinear dynamics," IEEE Trans. Cybern., vol. 43, no. 6, pp. 1688-1697, Dec. 2013.

[20] H. Su, M. Z. Q. Chen, X. Wang, and J. Lam, "Semiglobal observerbased leader-following consensus with input saturation," IEEE Trans. Ind. Electron., vol. 61, no. 6, pp. 2842-2850, Jun. 2014.

[21] G. Wen, W. Yu, Y. Xia, X. Yu, and J. Hu, "Distributed tracking of nonlinear multiagent systems under directed switching topology: An observer-based protocol," IEEE Trans. Syst., Man, Cybern., Syst., vol. 47, no. 5, pp. 869-881, May 2017.

[22] H. Li, Y. Gao, P. Shi, and H.-K. Lam, "Observer-based fault detection for nonlinear systems with sensor fault and limited communication capacity," IEEE Trans. Autom. Control, vol. 61, no. 9, pp. 2745-2751, Sep. 2016
[23] H. Li, P. Shi, D. Yao, and L. Wu, "Observer-based adaptive sliding mode control for nonlinear Markovian jump systems," Automatica, vol. 64, pp. 133-142, Feb. 2016.

[24] S. Tong, S. Sui, and Y. Li, "Observed-based adaptive fuzzy tracking control for switched nonlinear systems with dead-zone," IEEE Trans. Cybern., vol. 45, no. 12, pp. 2816-2826, Dec. 2015.

[25] S. Tong, L. Zhang, and Y. Li, "Observed-based adaptive fuzzy decentralized tracking control for switched uncertain nonlinear large-scale systems with dead zones," IEEE Trans. Syst., Man, Cybern., Syst., vol. 46, no. 1, pp. 37-47, Jan. 2016.

[26] H. Zhang and J. Wang, "Adaptive sliding-mode observer design for a selective catalytic reduction system of ground-vehicle diesel engines," IEEE/ASME Trans. Mechatronics, vol. 21, no. 4, pp. 2027-2038, Aug. 2016.

[27] H. Zhang, G. Zhang, and J. Wang, " $H_{\infty}$ observer design for LPV systems with uncertain measurements on scheduling variables: Application to an electric ground vehicle," IEEE/ASME Trans. Mechatronics, vol. 21, no. 3, pp. 1659-1670, Jun. 2016.

[28] X. Xie, D. Yue, and S. Hu, "Fault estimation observer design of discretetime nonlinear systems via a joint real-time scheduling law," IEEE Trans. Syst., Man, Cybern., Syst., vol. 47, no. 7, pp. 1451-1463, Jul. 2017.

[29] X. Xie, D. Yue, H. Zhang, and Y. Xue, "Fault estimation observer design for discrete-time Takagi-Sugeno fuzzy systems based on homogenous polynomially parameter-dependent Lyapunov functions," IEEE Trans. Cybern., vol. 47, no. 9, pp. 2504-2513, Sep. 2017.

[30] C. K. Ahn, P. Shi, and M. V. Basin, "Deadbeat dissipative FIR filtering," IEEE Trans. Circuits Syst. I, Reg. Papers, vol. 63, no. 8, pp. 1210-1221, Aug. 2016.

[31] H. Zhang, G. Feng, H. Yan, and Q. Chen, "Observer-based output feedback event-triggered control for consensus of multi-agent systems," IEEE Trans. Ind. Electron., vol. 61, no. 9, pp. 4885-4894, Sep. 2014.

[32] B. Zhou, C. Xu, and G. Duan, "Distributed and truncated reduced-order observer based output feedback consensus of multi-agent systems," IEEE Trans. Autom. Control, vol. 59, no. 8, pp. 2264-2270, Aug. 2014.

[33] L. Gao, Y. Cui, X. Xu, and Y. Zhao, "Distributed consensus protocol for leader-following multi-agent systems with functional observers," J. Frankl. Inst., vol. 352, no. 11, pp. 5173-5190, Nov. 2015.

[34] B. Kaviarasan, R. Sakthivel, and O.-M. Kwon, "Robust fault-tolerant control for power systems against mixed actuator failures," Nonlin. Anal. Hybrid Syst., vol. 22, pp. 249-261, Nov. 2016.

[35] H. Li, H. Gao, P. Shi, and X. Zhao, "Fault-tolerant control of Markovian jump stochastic systems via the augmented sliding mode observer approach," Automatica, vol. 50, no. 7, pp. 1825-1834, Jul. 2014.

[36] H. Li, W. Yan, and Y. Shi, "Continuous-time model predictive control of under-actuated spacecraft with bounded control torques," Automatica, vol. 75, pp. 144-153, Jan. 2017.

[37] M. Chen, P. Shi, and C.-C. Lim, "Adaptive neural fault-tolerant control of a 3-DOF model helicopter system," IEEE Trans. Syst., Man, Cybern., Syst., vol. 46, no. 2, pp. 260-270, Feb. 2016.

[38] S. Tong, S. Sui, and Y. Li, "Adaptive fuzzy decentralized tracking fault-tolerant control for stochastic nonlinear large-scale systems with unmodeled dynamics," Inf. Sci., vol. 289, pp. 225-240, Dec. 2014.

[39] H. Zhang and J. Wang, "Active steering actuator fault detection for an automatically-steered electric ground vehicle," IEEE Trans. Veh. Technol., vol. 66, no. 5, pp. 3685-3702, May 2017.

[40] Q. Zhou, H. Li, C. Wu, L. Wang, and C. K. Ahn, "Adaptive fuzzy control of nonlinear systems with unmodeled dynamics and input saturation using small-gain approach," IEEE Trans. Syst., Man, Cybern., Syst., vol. 47, no. 8, pp. 1979-1989, Aug. 2017, doi: 10.1109/TSMC.2016.2586108.

[41] B. Niu, C. K. Ahn, H. Li, and M. Liu, "Adaptive control for stochastic switched nonlower triangular nonlinear systems and its application to a one-link manipulator," IEEE Trans. Syst., Man, Cybern., Syst., to be published, doi: 10.1109/TSMC.2017.2685638.

[42] X. Wang and G.-H. Yang, "Distributed reliable $H_{\infty}$ consensus control for a class of multi-agent systems under switching networks: A topologybased average dwell time approach," Int. J. Robust Nonlin. Control, vol. 26, no. 13, pp. 2767-2787, Sep. 2016.

[43] S. Chen, D. W. C. Ho, L. Li, and M. Liu, "Fault-tolerant consensus of multi-agent system with distributed adaptive protocol," IEEE Trans. Cybern., vol. 45, no. 10, pp. 2142-2155, Oct. 2015.

[44] J. Lin, Y. Shi, S. Fei, and Z. Gao, "Reliable dissipative control of discrete-time switched singular systems with mixed time delays and stochastic actuator failures," IET Control Theory Appl., vol. 7, no. 11, pp. 1447-1462, Jul. 2013. 
[45] S. Wang, J. Feng, and H. Zhang, "Robust fault tolerant control for a class of networked control systems with state delay and stochastic actuator failures," Int. J. Adapt. Control Signal Process., vol. 28, no. 9, pp. 798-811, Sep. 2014.

[46] C. K. Ahn, L. Wu, and P. Shi, "Stochastic stability analysis for 2-D Roesser systems with multiplicative noise," Automatica, vol. 69, pp. 356-363, Jul. 2016.

[47] C. K. Ahn, P. Shi, and L. Wu, "Receding horizon stabilization and disturbance attenuation for neural networks with time-varying delay," IEEE Trans. Cybern., vol. 45, no. 12, pp. 2680-2692, Dec. 2015.

[48] H. Yanai, K. Takeuchi, and Y. Takane, Projection Matrices, Generalized Inverse Matrices, and Singular Value Decomposition. New York, NY, USA: Springer-Verlag, 2011.

[49] L. V. Hien and H. Trinh, "Refined Jensen-based inequality approach to stability analysis of time-delay systems," IET Control Theory Appl., vol. 9, no. 14, pp. 2188-2194, Sep. 2015.

[50] C. K. Ahn, P. Shi, and M. V. Basin, "Two-dimensional dissipative control and filtering for Roesser model," IEEE Trans. Autom. Control, vol. 60 no. 7, pp. 1745-1759, Jul. 2015. 